\title{
On the structure of Leibniz algebras whose subalgebras are ideals or core-free
}

\author{
V. A. Chupordia, L. A. Kurdachenko, and N. N. Semko
}

To Igor Subbotin on the occasion of his 70th birthday

A Bstract. An algebra $L$ over a field $F$ is said to be a Leibniz algebra (more precisely, a left Leibniz algebra) if it satisfies the Leibniz identity: $[[a, b], c]=[a,[b, c]]-[b,[a, c]]$ for all $a, b, c \in L$. Leibniz algebras are generalizations of Lie algebras. A subalgebra $S$ of a Leibniz algebra $L$ is called a core-free, if $S$ does not include a non-zero ideal. We study the Leibniz algebras, whose subalgebras are either ideals or core-free.

\section{Introduction}

Let $L$ be an algebra over a field $F$ with the binary operations + and $[\cdot, \cdot]$. Then $L$ is called a Leibniz algebra (more precisely, a left Leibniz algebra), if it satisfies the Leibniz identity

$$
[a,[b, c]]=[[a, b], c]+[b,[a, c]],
$$

for all $a, b, c \in L$.

If $L$ is a Lie algebra, then $L$ is a Leibniz algebra. Conversely, if $L$ is a Leibniz algebra such that $[a, a]=0$ for every element $a \in L$, then $L$ is a Lie algebra. Therefore, Lie algebras can be characterized as the Leibniz algebras in which $[a, a]=0$ for every element $a$.

Leibniz algebras appeared first in the papers by A.M. Bloh [1-3] in which he called them the D-algebras. However, in that time, these works

2010 MSC: 17A32, 17A60, 17A99.

Key words and phrases: Leibniz algebra, Lie algebra, ideal, core-free subalgebras, monolithic algebra, extraspecial algebra. 
were not in demand, and they had not been properly developed. Only in two decades, a real interest in Leibniz algebras rose. It is happened due to the work of by J.L. Loday [4] (see also [5, Section 10.6]), who "rediscovered" these algebras and used the term Leibniz algebras, since it was Leibniz, who discovered and proved the Leibniz rule for the differentiation of functions.

A Leibniz algebra which is not a Lie algebra has one specific ideal. Denote, by Leib $(L)$, the subspace generated by the elements $[a, a], a \in L$. It is possible to prove that $\operatorname{Leib}(L)$ is an ideal of $L$. Moreover, $L / \operatorname{Leib}(L)$ is a Lie algebra. Conversely, if $H$ is an ideal of $L$ such that $L / H$ is a Lie algebra, then $\operatorname{Leib}(L) \leqslant H$.

The ideal Leib $(L)$ is called the Leibniz kernel of the algebra $L$.

One approach to the study of Leibniz algebras, which proved to be quite efficient especially for infinite dimensional Leibniz algebras, is to consider Leibniz algebras, all whose subalgebras have some fixed natural properties. This approach has been very efficient for Lie algebras, while it became to be used in Leibniz algebras only recently. The Leibniz algebras, whose subalgebras are Lie algebras and Leibniz algebras with Abelian subalgebras, were studied in [6]. In paper [7] (see also [8]), the Leibniz algebras, whose subalgebras are ideals, were studied.

Two ideals are naturally associated with each subalgebra $A$ of a Leibniz algebra $L$ : the ideal $A^{L}$ which is the intersection of all ideals including $A$ (i.e., an ideal generated by $A$ ); and the ideal $\operatorname{Core}_{L}(A)$ which is the sum of all ideals that are contained in $A$.

A subalgebra $A$ of $L$ is called a core-free in $L$ if $\operatorname{Core}_{L}(A)=\langle 0\rangle$. From the definition, it follows that the core-free subalgebras are natural antipodes to the concepts of ideals. Therefore, the study of Leibniz algebras, whose subalgebras are either core-free or ideals, seems to us very natural. The main results of the current article give a description of such Leibniz algebras.

The first example of such algebras is Leibniz algebras whose subalgebras are ideals. As we have noted above, these Leibniz algebras have been studied in [7]. The main result of this paper is as follows:

If every subalgebra of a Leibniz algebra $L$ is an ideal, then either $L$ is Abelian or $L=Z \oplus E$ where $Z \leqslant \zeta(L)$ and $E$ is an extraspecial subalgebra such that $[a, a] \neq 0$ for each element $a \in E \backslash \zeta(L)$.

In particular, $[L, L] \leqslant \zeta(L)$, and a derived subalgebra $[L, L]$ has dimension 1.

Recall that a Leibniz algebra $E$ is called extraspecial, if $\zeta(E)=[E, E]$ is a subalgebra of dimension 1 .

Note also that a Lie algebra, whose subalgebras are ideals, is Abelian. 
On the other hand, if $L$ is a simple Leibniz algebra, then every its proper subalgebra is core-free. We note that, in this case, $L$ is a Lie algebra.

We show also another example which is typical in some sense. Let $L$ be a cyclic nilpotent Leibniz algebra of dimension 3. That is, $L=F a \oplus F b \oplus F c$, where $b=[a, a], c=[a, b]$. Here, $\operatorname{Leib}(L)=F b \oplus F c$. If $A$ is a subalgebra of $L$ such that Leib $(L)$ does not include $A$, then $A=L$. If $A \leqslant \operatorname{Leib}(L)$ and $F c \leqslant A$, then $A$ is an ideal of $L$. If $A$ does not include $F c$, then $A$ is not an ideal, $\operatorname{dim}_{F}(A)=1$. Therefore, $\operatorname{Core}_{L}(A)=\langle 0\rangle$.

Let $L$ be a Leibniz algebra. The intersection of all non-zero ideals $\operatorname{Mon}(L)$ of $L$ is called the monolith of a Leibniz algebra $L$. If $\operatorname{Mon}(L) \neq\langle 0\rangle$, then the Leibniz algebra $L$ is called monolithic, and, in this case, $\operatorname{Mon}(G)$ is the least non-zero ideal of $L$.

The next proposition shows how the Leibniz algebras, whose subalgebras being not ideals are core-free, appear.

Proposition 1. Let $L$ be a monolithic Leibniz algebra. If every subalgebra of a factor-algebra $L / \operatorname{Mon}(L)$ is an ideal, then every subalgebra of $L$ are either core-free or ideal.

Indeed, let $A$ be a subalgebra of $L$ such that $B=\operatorname{Core}_{L}(A) \neq\langle 0\rangle$. Since $L$ is monolithic, $\operatorname{Mon}(L) \leqslant B \leqslant A$. Since every subalgebra of $L / \operatorname{Mon}(L)$ is an ideal, $A$ is an ideal of $L$.

Thus, we can see that the following natural cases hold:

- $L$ is a non-monolithic Leibniz algebra;

- $L$ is a monolithic Leibniz algebra.

The second case is basic, as is shown by

Theorem 1. Let $L$ be a non-monolithic Leibniz algebra. If every subalgebra of $L$, which is not an ideal, is core-free, then every subalgebra of $L$ is an ideal.

Corollary 1. Let $L$ be a non-monolithic Lie algebra. If every subalgebra of $L$, which is not an ideal, is core-free, then $L$ is Abelian.

The monolithic case splits naturally in two subcases:

- Leibniz algebra $L$ has a non-zero center;

- Leibniz algebra $L$ has a zero center.

Recall that the center $\zeta(L)$ of $L$ is defined by the rule:

$$
\zeta(L)=\{x \in L \mid[x, y]=0=[y, x] \text { for every element } y \in L\} .
$$

The center is an ideal of $L$. In particular, we can consider the factor-algebra $L / \zeta(L)$. 
More precisely, the left (respectively, right) center $\zeta^{\text {left }}(L)$ (respectively, $\left.\zeta^{\text {right }}(L)\right)$ of a Leibniz algebra $L$ is defined by the rule:

$$
\zeta^{\text {left }}(L)=\{x \in L \mid[x, y]=0 \text { for every element } y \in L\} .
$$

(respectively,

$$
\left.\zeta^{\text {right }}(L)=\{x \in L \mid[y, x]=0 \text { for every element } y \in L\} .\right)
$$

It is not hard to prove that the left center of $L$ is an ideal, but it is not true for the right center. Moreover, $\operatorname{Leib}(L) \leqslant \zeta^{\text {left }}(L)$, so that $L / \zeta^{\text {left }}(L)$ is a Lie algebra. The right center is an subalgebra of $L$, and, in general, the left and right centers are different; they even may have different dimensions. Paper [9] contains some examples, which are shown by

Theorem 2. Let $L$ be a Leibniz algebra. Suppose that $L$ includes a subalgebra, which is not an ideal, and every subalgebra of L, which is not an ideal, is core-free. If the center of $L$ is non-zero, then $L$ satisfies the following conditions:

(i) $L$ is monolithic, and $\operatorname{Mon}(L)=\zeta(L)=\gamma_{3}(L)$, in particular, $\operatorname{dim}_{F}(\zeta(L))=1$

(ii) $\gamma_{2}(L)=[L, L] \leqslant \zeta_{2}(L)$ and $\gamma_{2}(L)$ has dimension 2;

(iii) every subalgebra of $L$, which is not an ideal, is Abelian;

(iv) every subalgebra of $L / \zeta(L)$ is an ideal.

Conversely, if $L$ is a Leibniz algebra satisfying the above conditions, then every subalgebra of $L$ either is core-free or an ideal.

Corollary 2. Let $L$ be a monolithic non-Abelian Lie algebra having nontrivial center. Then every subalgebra of $L$, which is not an ideal, is core-free, if and only if $L$ is an extraspecial algebra.

The situation, where $L$ has a non-central monolith, was considered in the following proposition.

Theorem 3. Let $L$ be a monolithic Leibniz algebra whose center is zero. Suppose that every subalgebra of $L$, which is not an ideal, is core-free. If $L$ is not a Lie algebra, then the following conditions hold:

(i) $\operatorname{Mon}(L)$ is a minimal ideal of $L$;

(ii) $\operatorname{Mon}(L)$ is a maximal Abelian ideal of $L$;

(iii) $L=\operatorname{Mon}(L) \oplus A$ for some Abelian subalgebra $A$;

(iv) $\operatorname{Ann}_{L}(\operatorname{Mon}(L))=\operatorname{Ann}_{L}^{\operatorname{left}}(\operatorname{Mon}(L))=\operatorname{Mon}(L)$.

Conversely, if $L$ is a Leibniz algebra satisfying the above conditions, then every subalgebra of $L$ either is core-free or an ideal. 
We note also that, in this case, a core-free subalgebra can be not Abelian. The following example shows this.

Example 1. Let $F$ be an arbitrary field, and let $L$ be a vector space over $F$ with a basis $a, b, a_{1}, a_{2}$. We define the operation $[\cdot, \cdot]$ on $L$ in the following way:

$$
\begin{gathered}
{[a, a]=a_{1}, \quad\left[a, a_{1}\right]=a_{2}, \quad\left[a, a_{2}\right]=-a_{1}-a_{2}, \quad[a, b]=0,} \\
{[b, a]=a_{1}+a_{2}, \quad[b, b]=0, \quad\left[b, a_{1}\right]=-a_{1}, \quad\left[b, a_{2}\right]=-a_{2},} \\
{\left[a_{1}, a\right]=0, \quad\left[a_{1}, b\right]=0, \quad\left[a_{2}, a\right]=0, \quad\left[a_{2}, b\right]=0,} \\
{\left[a_{1}, a_{1}\right]=0, \quad\left[a_{1}, a_{2}\right]=0, \quad\left[a_{2}, a_{1}\right]=0, \quad\left[a_{2}, a_{2}\right]=0 .}
\end{gathered}
$$

It is possible to check that $L$ is a Leibniz algebra, $\operatorname{Leib}(L)=F a_{1}+$ $F a_{2}, \operatorname{Leib}(L)=\operatorname{Mon}(L)$, and a factor-algebra $L / \operatorname{Mon}(L)$ is Abelian. Proposition 1 implies that every subalgebra of $L$, which is not an ideal, is core-free. But the subalgebra $\left\langle b, a_{1}\right\rangle$ is a not ideal, is not Abelian, and is core-free.

For the Lie algebras, we obtained the following

Proposition 2. Let $L$ be a monolithic Lie algebra whose center is zero. Suppose that every subalgebra of $L$, which is not an ideal, is core-free. Then $\operatorname{Mon}(L)$ is a minimal ideal of $L$ such that $\operatorname{Ann}_{L}(\operatorname{Mon}(L))=\operatorname{Mon}(L)$ and the factor-algebra $L / \operatorname{Mon}(L)$ is Abelian. Moreover, every core-free subalgebra of $L$ is Abelian.

If $\operatorname{Mon}(L)$ is Abelian, the description should be more detailed.

Let $L$ be a Leibniz algebra, and let $a$ be a some fixed element of $L$. Consider the mapping $\mathbf{r}_{a}: L \mapsto L$ defined by the rule $\mathbf{r}_{a}(x)=[x, a]$, $x \in L$. It is not hard to see that $\mathbf{r}_{a}$ is a linear mapping, $\beta \mathbf{r}_{a}=\mathbf{r}_{\beta a}$, and $\mathbf{r}_{a}+\mathbf{r}_{b}=\mathbf{r}_{a+b}$ for all $a, b \in L$ and $\beta \in F$. Put $\mathbf{c}_{a}(x)=x+[x, a], x \in L$, i.e., $\mathbf{c}_{a}(x)=\mathbf{i}(x)+\mathbf{r}_{a}(x)$, where $\mathbf{i}$ is an identity permutation of $L$. Clearly, $\mathbf{c}_{a}$ is also a linear mapping.

Theorem 4. Let $L$ be a monolithic Lie algebra whose center is zero. Suppose that every subalgebra of $L$, which is not an ideal, is core-free. If the monolith of $L$ is Abelian, then the following conditions hold:

(i) $\operatorname{Mon}(L)$ is a minimal ideal of $L$;

(ii) $\operatorname{Mon}(L)$ is a maximal Abelian ideal of $L$;

(iii) $\operatorname{Ann}_{L}(\operatorname{Mon}(L))=\operatorname{Mon}(L)$;

(iv) $L=\operatorname{Mon}(L) \oplus A$ for some Abelian subalgebra $A$;

(v) if $L=\operatorname{Mon}(L) \oplus C$ for some subalgebra $C$, then there exists an element $v \in \operatorname{Mon}(L)$ such that $C=\boldsymbol{c}_{v}(A)$. Moreover, $\boldsymbol{c}_{v}$ is an automorphism of the algebra $L$. 


\section{Preliminary results}

We start from the following simple result.

Lemma 1. Let $L$ be a Leibniz algebra, and let $A$ be a non-zero ideal of $L$. If every subalgebra of $L$, which is not an ideal, is core-free, then every subalgebra of $L / A$ is an ideal.

Proof. Indeed, let $x$ be an element of $L$ such that $x \notin A$. We denote, by $X$, the subalgebra of $L$ generated by $x$ and $A$. Then $X$ includes a non-zero ideal $A$. It follows that $\operatorname{Core}_{L}(X) \neq\langle 0\rangle$. Hence, $X$ must be an ideal of $L$. This means that every cyclic subalgebra of $L / A$ is an ideal. Then each subalgebra of $L / A$ is an ideal of $L / A$.

Let $L$ be a Lie algebra. We define the lower central series of $L$,

$$
L=\gamma_{1}(L) \geqslant \gamma_{2}(L) \geqslant \ldots \geqslant \gamma_{\alpha}(L) \geqslant \gamma_{\alpha+1}(L) \geqslant \ldots \gamma_{\delta}(L),
$$

by the following rule: $\gamma_{1}(L)=L, \gamma_{2}(L)=[L, L]$, and, recursively, $\gamma_{\alpha+1}(L)=\left[L, \gamma_{\alpha}(L)\right]$ for all ordinals $\alpha$, and $\gamma_{\lambda}(L)=\bigcap_{\mu<\lambda} \gamma_{\mu}(L)$ for the limit ordinals $\lambda$. The last term $\gamma_{\delta}(L)$ is called the lower hypocenter of $L$. We have $\gamma_{\delta}(L)=\left[L, \gamma_{\delta}(L)\right]$.

If $\alpha=k$ is a positive integer, then $\gamma_{k}(L)=[L,[L,[L, \ldots, L] \ldots]$ is the left normed commutator of $k$ copies of $L$. Note the following useful properties of subalgebras and ideals.

Corollary 3. Let $L$ be a Leibniz algebra, and let $A$ be a non-zero ideal of $L$. If every subalgebra of $L$, which is not an ideal, is core-free, then $\gamma_{3}(L) \leqslant A$.

Proof. Indeed, as we have noted above, a Leibniz algebra, whose subalgebras are ideals, is Abelian or nilpotent and belongs to the 2-nilpotency class.

Corollary 4. Let $L$ be a non-monolithic Leibniz algebra. If every subalgebra of $L$, which is not an ideal, is core-free, then $\gamma_{3}(L)=\langle 0\rangle$.

Proof. Indeed, let $\mathfrak{S}$ be a family of all non-zero ideals of $L$. Then Corollary 3 implies that $\gamma_{3}(L) \leqslant S$ for each non-zero ideal $S$ of $L$. Since $L$ is non-monolithic, $\cap \mathfrak{S}=\langle 0\rangle$. It follows that $\gamma_{3}(L)=\langle 0\rangle$.

Corollary 5. Let $L$ be a non-monolithic Leibniz algebra. If every subalgebra of $L$, which is not an ideal, is core-free, then its center is non-zero. 
Lemma 2. Let $L$ be a Leibniz algebra whose center is non-zero. Suppose that every subalgebra of $L$, which is not an ideal, is core-free. If $C$ is a non-zero core-free subalgebra of $L$, then $C$ is Abelian.

Proof. Since $C$ is core-free, then $C \cap \zeta(L)=\langle 0\rangle$. Assume that $C$ contains a non-zero element $c$ such that $c_{1}=[c, c] \neq 0$. Using Corollary 3 of Lemma 1 , we obtain that $[c,[c, c]] \in \zeta(L)$. On the other hand, since $C$ is a subalgebra, $[c,[c, c]] \in C$, so that $[c,[c, c]] \in C \cap \zeta(L)=\langle 0\rangle$. It follows that $\langle c\rangle=F c \oplus F c_{1}$. The fact that $C$ is core-free implies that every its subalgebra is core-free. It follows that the subalgebra $\left\langle c_{1}\right\rangle=F c_{1}$ is not an ideal of $L$. Then there exists an element $x$ such that $\left[x, c_{1}\right] \notin\left\langle c_{1}\right\rangle$. By Lemma 1, a subalgebra $\left\langle c_{1}, \zeta(L)\right\rangle=\left\langle c_{1}\right\rangle \oplus \zeta(L)=F c_{1} \oplus \zeta(L)$ is an ideal of $L$. This implies that $\left[x, c_{1}\right] \in F c_{1} \oplus \zeta(L)$, i.e., $\left[x, c_{1}\right]=\alpha c_{1}+z$, where $\alpha \in F, z \in \zeta(L)$. Clearly, $z \neq 0$. Using again Lemma 1 , we obtain that the subalgebra $\langle c, \zeta(L)\rangle=\langle c\rangle \oplus \zeta(L)=F c \oplus F c_{1} \oplus \zeta(L)$ is an ideal of $L$. Then $[x, c]=\beta c+\gamma c_{1}+z_{1}$, where $\beta, \gamma \in F, z_{1} \in \zeta(L)$. We have

$$
[[x, c], c]=\left[\beta c+\gamma c_{1}+z_{1}, c\right]=\beta c_{1} .
$$

On the other hand,

$$
\begin{aligned}
{[[x, c], c] } & =[x,[c, c]]-[c,[x, c]]=\left[x, c_{1}\right]-\left[c, \beta c+\gamma c_{1}+z_{1}\right] \\
& =\alpha c_{1}+z-\beta c_{1}=(\alpha-\beta) c_{1}+z .
\end{aligned}
$$

Thus, we obtain $\beta c_{1}=(\alpha-\beta) c_{1}+z$ or $(\alpha-2 \beta) c_{1}=z$. By the above consideration, the element $z$ is non-zero, so that $(\alpha-2 \beta) c_{1}$ is a non-zero element of $\zeta(L)$. But this contradicts the equality $C \cap \zeta(L)=\langle 0\rangle$. This contradiction shows that $[y, y]=0$ for each element $y \in C$. It follows that $\langle y\rangle=F y$ for each element $y \in C$.

If $\operatorname{dim}_{F}(C)=1$, all is proved. Therefore, we will suppose that $\operatorname{dim}_{F}(C)>1$. Let $\left\{c_{\lambda} \mid \lambda \in \Lambda\right\}$ be the basis of $C$. By the above proof, $\left\langle c_{\lambda}\right\rangle=F c_{\lambda}$. Lemma 1 shows that $\left\langle c_{\lambda}, \zeta(L)\right\rangle=F c_{\lambda} \oplus \zeta(L)$ is an ideal of $L$. It follows that $\left[c_{\lambda}, c_{\mu}\right] \in F c_{\lambda} \oplus \zeta(L)$, i.e., $\left[c_{\lambda}, c_{\mu}\right]=\sigma c_{\lambda}+z_{2}$ for some elements $\sigma \in F, z_{2} \in \zeta(L)$. By the same reason, $\left[c_{\lambda}, c_{\mu}\right]=\tau c_{\mu}+z_{3}$ for some elements $\tau \in F, z_{3} \in \zeta(L)$. Thus, we have $\sigma c_{\lambda}-\tau c_{\mu}=z_{2}-z_{3} \in C \cap \zeta(L)=\langle 0\rangle$. The fact that the elements $c_{\lambda}, c_{\mu}$ are linearly independent implies that $\sigma=\tau=0$. This means that $\left[c_{\lambda}, c_{\mu}\right] \in \zeta(L)$. On the other hand, since $C$ is a subalgebra, $\left[c_{\lambda}, c_{\mu}\right] \in C$, so that $\left[c_{\lambda}, c_{\mu}\right] \in C \cap \zeta(L)=\langle 0\rangle$. It is true for each pair of indices $\lambda, \mu$, and it follows that a subalgebra $C$ is Abelian. 


\section{Proof of Theorem 1}

Let $\mathfrak{S}$ be a family of all non-zero ideals of $L$. Since $L$ is non-monolithic, $\cap \mathfrak{S}=\langle 0\rangle$. Moreover, Corollary 4 of Lemma 1 implies that $\gamma_{3}(L)=\langle 0\rangle$. In particular, $\zeta(L)$ is non-zero. If every cyclic subalgebra of $L$ is an ideal, then every subalgebra of $L$ is an ideal. Therefore, we assume that $L$ includes a cyclic subalgebra $\langle a\rangle$, which is not an ideal. Then $\langle a\rangle$ must be core-free. Lemma 2 implies that $\langle a\rangle$ is Abelian, and, therefore, $\langle a\rangle=F a$. In particular, $\langle a\rangle$ has dimension 1 . Lemma 1 implies that a subalgebra $(\langle a\rangle+S) / S$ of the factor-algebra $L / S$ is an ideal of $L / S$ for each ideal $S \in \mathfrak{S}$. Since $L / S$ is nilpotent and ideal, $(\langle a\rangle+S) / S$ has dimension 1. Lemma 2.4 in [10] implies that $(\langle a\rangle+S) / S \leqslant \zeta(L / S)$ for each ideal $S \in \mathfrak{S}$. Then $[x, a],[a, x] \in S$ for every element $x \in L$. Since it is true for each $S \in \mathfrak{S},[x, a],[a, x] \in \cap \mathfrak{S}=\langle 0\rangle$. This means that $a \in \zeta(L)$, that follows from the fact that a subalgebra $\langle a\rangle$ is an ideal of $L$. We have obtained a contradiction. This contradiction proves that every cyclic subalgebra of $L$ is an ideal.

Corollary 6. Let $L$ be a Leibniz algebra. Suppose that every subalgebra of $L$, which is not an ideal, is core-free. If the center of $L$ is non-zero and $\operatorname{dim}_{F}(\zeta(L))>1$, then every subalgebra of $L$ is an ideal.

Proof. Since $\operatorname{dim}_{F}(\zeta(L))>1, \zeta(L)$ includes two non-zero subspaces $Z_{1}$, $Z_{2}$ such that $Z_{1} \cap Z_{2}=\langle 0\rangle$. It follows that $L$ is not monolithic, and we can apply Theorem 1.

\section{Proof of Theorem 2}

If we suppose that $\operatorname{dim}_{F}(\zeta(L))>1$, then Corollary 6 shows that every subalgebra of $L$ is an ideal, and we obtain a contradiction. This contradiction shows that $\operatorname{dim}_{F}(\zeta(L))=1$. Corollary 6 shows that $L$ must be monolithic. An obvious inclusion $\operatorname{Mon}(L) \leqslant \zeta(L)$ together with the fact that $\operatorname{dim}_{F}(\zeta(L))=1$ imply that $\operatorname{Mon}(L)=\zeta(L)$. Lemma 1 shows that every subalgebra of $L / \zeta(L)$ is an ideal. Using now Theorem A in [7], we obtain that $\zeta(L / \zeta(L))$ includes $[L / \zeta(L), L / \zeta(L)]$ and $[L / \zeta(L), L / \zeta(L)]$ has dimension 1. Furthermore, the equality $\operatorname{Mon}(L)=\zeta(L)$ implies that $\zeta(L) \leqslant[L, L]$, and the equality $[L / \zeta(L), L / \zeta(L)]=[L, L] \zeta(L) / \zeta(L)$ implies that $[L / \zeta(L), L / \zeta(L)]=[L, L] / \zeta(L)$, so that $[L, L]$ has dimension 2, and $\zeta(L / \zeta(L)) \geqslant[L, L] / \zeta(L)$. Therefore, $\zeta_{2}(L) \geqslant[L, L]=\gamma_{2}(L)$. Condition (iii) follows from Lemma 2 , and condition (iv) follows from Lemma 1. 
Conversely, let $L$ be a Leibniz algebra satisfying conditions (i) - (iv). Then conditions (i) and (iv) together with Proposition 1 imply that every subalgebra of $L$, which is not an ideal, is core-free.

Corollary 7. Let $L$ be a Leibniz algebra. Suppose that $L$ includes a subalgebra, which is not an ideal, and every subalgebra of $L$, which is not an ideal, is core-free. If $\gamma_{3}(L)=\langle 0\rangle$, then $L$ is an extraspecial Leibniz algebra. Conversely, if $L$ is an extraspecial Leibniz algebra, then every subalgebra of $L$, which is not an ideal, is core-free.

Proof. Since $\gamma_{3}(L)=\langle 0\rangle$, a factor-algebra $L / \zeta(L)$ is Abelian. The fact that $L$ includes a subalgebra, which is not an ideal, implies that $L$ is nonAbelian. In turn, this implies that $L \neq \zeta(L)$ and $[L, L] \neq\langle 0\rangle$. Corollary 3 implies that $\operatorname{dim}_{F}(\zeta(L))=1$. Since $[L, L] \neq\langle 0\rangle$ and $[L, L] \leqslant \zeta(L)$, $[L, L]=\zeta(L)$. This means that $L$ is an extraspecial Leibniz algebra.

Conversely, let $L$ be an extraspecial Leibniz algebra, and let $A$ be a subalgebra of $L$ such that $B=\operatorname{Core}_{L}(A) \neq\langle 0\rangle$. Since $L$ is nilpotent, Lemma 2.4 in [10] implies that $B \cap \zeta(L) \neq\langle 0\rangle$. The fact that $\operatorname{dim}_{F}(\zeta(L))=$ 1 implies that $\zeta(L) \leqslant B \leqslant A$. For each arbitrary subalgebra $D$, we have $\zeta(L) \leqslant D$ or $D \cap \zeta(L)=\langle 0\rangle$. In the first case, $D$ is an ideal. In the second one, in view of the above consideration, we obtain that $D$ is core-free.

Lemma 3. Let $L$ be a Lie algebra, whose subalgebras are ideals. Then $L$ is Abelian.

This lemma is almost obvious.

\section{Proof of Corollary 2}

As above, we obtain that $\operatorname{Mon}(L)=\zeta(L)$ and $\operatorname{dim}_{F}(\zeta(L))=1$. Lemma 1 shows that every subalgebra of $L / \zeta(L)$ is an ideal. Then Lemma 3 implies that a factor-algebra $L / \zeta(L)$ is Abelian. It follows that $[L, L] \leqslant \zeta(L)$. Since $L$ is not Abelian, the equality $\operatorname{dim}_{F}(\zeta(L))=1$ implies that $[L, L]=\zeta(L)$, so that $L$ is an extraspecial Leibniz algebra.

Lemma 4. Let $L$ be a monolithic Leibniz algebra whose center is zero. Suppose that every subalgebra of $L$, which is not an ideal, is core-free. If $L$ is not a Lie algebra, then $\operatorname{Mon}(L)$ is a minimal ideal of $L$ and a maximal Abelian ideal of $L$, and the factor-algebra $L / \operatorname{Mon}(L)$ is Abelian.

Proof. Since $L$ is not Lie algebra, its Leibniz kernel Leib $(L)$ is non-zero. Recall that the left center of $L$ includes $\operatorname{Leib}(L)$. It follows that Leib $(L)$ is 
Abelian. The inclusion $M=\operatorname{Mon}(L) \leqslant \operatorname{Leib}(L)$ implies that $M$ is Abelian. The factor-algebra $L / \operatorname{Leib}(L)$ is a Lie algebra. Lemma 1 shows that every subalgebra of this factor-algebra must be an ideal. Using Lemma 3, we obtain that $L / \operatorname{Leib}(L)$ is Abelian. Since $\zeta(L)=\langle 0\rangle, L \neq \operatorname{Ann}_{L}(M)$.

Let $A$ be a maximal Abelian ideal of $L$ including $\operatorname{Leib}(L)$. Suppose that $A \neq M$. The application of Lemma 1 shows that every subalgebra of $L / M$ is an ideal. Theorem A in [7] shows that $L / M$ is nilpotent. It follows that $A$ has a finite series

$$
\langle 0\rangle=M_{0} \leqslant M=M_{1} \leqslant M_{2} \leqslant M_{3}=A
$$

of $L$-invariant subalgebras, where the factors $M_{3} / M_{2}$ and $M_{2} / M_{1}$ are $L$-central. Using Proposition 1.3 in [11], we obtain that $A$ includes an $L$ invariant subalgebra $D$ such that $A=M \oplus D$. In particular, $D \cap M=\langle 0\rangle$. On the other hand, $L$ is a monolithic Leibniz algebra, and we obtain a contradiction. This contradiction shows that $A=M$.

Lemma 5. Let $L$ be a monolithic Leibniz algebra whose center is zero. Suppose that every subalgebra of $L$, which is not an ideal, is core-free. If $L$ is not a Lie algebra, then $L=\operatorname{Mon}(L) \oplus A$ for some Abelian subalgebra A. Moreover, $\operatorname{Ann}_{L}(\operatorname{Mon}(L))=\operatorname{Ann}_{L}^{\text {left }}(\operatorname{Mon}(L))=\operatorname{Mon}(L)$.

Proof. By its definition, $M=\operatorname{Mon}(L)$ is a minimal ideal of $L$. Lemma 4 shows that the factor-algebra $L / M$ is Abelian. It follows that $[x, y] \in M$ for every pairs of elements $x, y \in L$. The inclusion $\operatorname{Mon}(L) \leqslant \operatorname{Leib}(L)$ and the fact that the left center of $L$ includes $\operatorname{Leib}(L)$ yield $[M, L]=\langle 0\rangle$. It follows that $\operatorname{Ann}_{L}(M)=\operatorname{Ann}_{L}^{\text {left }}(M)$. Since $\langle 0\rangle=M \cap \zeta(L)$, there exists an element $d$ such that $d \notin \operatorname{Ann}_{L}(M)$. Put

$$
[d, M]=\{[d, a] \mid a \in M\} .
$$

Clearly, $[d, M]$ is a subspace of $M$. Since $M$ is Abelian, $[d, M]$ is a subalgebra of $L$. Let $x$ be an arbitrary element of $L$. For each element $a \in A$, we have

$$
[x,[d, a]]=[[x, d], a]+[d,[x, a]] .
$$

By the above remark, $[x, d] \in M$. Therefore, $[[x, d], a]=0$, because $M$ is Abelian. Since $M$ is an ideal, $[x, a] \in M$, so that $[d,[x, a]] \in[d, M]$ and $[x,[d, a]] \in M$. Further, we have

$$
[[d, a], x]=[d,[a, x]]-[a,[d, x]] .
$$


Again, $[a,[d, x]]=0$ and $[a, x] \in M$. Therefore, $[d,[a, x]] \in[d, M]$ and $[[d, a], x] \in M$. This means that $[d, M]$ is an ideal of $L$. The choice of $d$ shows that $[d, M]$ is a non-zero ideal. Since $M$ is a minimal ideal, we obtain that $M=[d, M]$.

Let again $x$ be an arbitrary element of $L$. As we noted above, $[d, x] \in M$. The equality $M=[d, M]$ shows that there exists an element $b \in M$ such that $[d, x]=[d, b]$. It follows that $[d, x-b]=0$, so that $x-b=c \in$ $\operatorname{Ann}_{L}^{\text {right }}(d)$. Then $x=b+c \in M+A$, where $A=\operatorname{Ann}_{L}^{\text {right }}(d)$.

Let $a \in A \cap M=\operatorname{Ann}_{M}^{\text {right }}(d)$. For an arbitrary element $x \in L$, we have

$$
[d,[a, x]]=[[d, a], x]+[a,[d, x]] .
$$

The choice of $a$ shows that $[d, a]=0$. Since $a \in M \leqslant \operatorname{Leib}(L) \leqslant \zeta^{\text {left }}(L)$, $[a,[d, x]]=0$, so that $[d,[a, x]]=0$. Since $[a, x]=0,[[a, x], d]=0$. This means that $\operatorname{Ann}_{M}^{\text {right }}(d)$ is an ideal of $L$. The fact that $M$ is a minimal ideal implies that either $\operatorname{Ann}_{M}^{\text {right }}(d)=M$ or $\operatorname{Ann}_{M}^{\text {right }}(d)=\langle 0\rangle$. The choice of an element $d$ shows that the equality $\operatorname{Ann}_{M}^{\text {right }}(d)=M$ is impossible, so that $\langle 0\rangle=\operatorname{Ann}_{M}^{\text {right }}(d)=A \cap M$.

Finally, suppose that $\operatorname{Ann}_{L}^{\text {left }}(M)=K \neq M$. We have noted above that $\operatorname{Ann}_{L}^{\text {left }}(M)=\operatorname{Ann}_{L}(M)$. It follows that $K$ is an ideal of $L$. The obvious inclusion $M \leqslant K$ implies that $K=M \oplus(K \cap A)$. Being a subalgebra of an Abelian algebra $A, K \cap A$ is also Abelian. If $u, v$ be an arbitrary elements of $K$, then $u=a_{1}+c_{1}, v=a_{2}+c_{2}$, where $a_{1}, a_{2} \in M, c_{1}, c_{2} \in K \cap A$. We have

$$
[u, v]=\left[a_{1}+c_{1}, a_{2}+c_{2}\right]=\left[a_{1}, a_{2}\right]+\left[a_{1}, c_{2}\right]+\left[c_{1}, a_{2}\right]+\left[c_{1}, c_{2}\right]=0 .
$$

Then an ideal $K$ is Abelian. On the other hand, Lemma 4 shows that $M$ is a maximal Abelian ideal of $L$, and we obtain a contradiction. This contradiction proves that $\operatorname{Ann}_{L}^{\text {left }}(M)=M$.

\section{Proof of Theorem 3}

Conditions (i), (ii) follow from Lemma 4. Conditions (iii), (vi) follow from Lemma 5.

\section{Proof of Proposition 2}

Let $M$ be a monolith of $L$. By its choice, $M$ is a minimal ideal of $L$. By Lemma 1, every subalgebra of $L / M$ is an ideal of $L / M$. Lemma 3 shows that $L / M$ must be Abelian. 
Suppose that $A=\operatorname{Ann}_{L}(M) \neq M$. Consider firstly the case where $M$ is Abelian. Choose an element $d \in A \backslash M$. Put $D=\langle a, M\rangle$. Since $a$ factoralgebra $L / M$ is Abelian, $D$ is an ideal of $L$. The fact that $d \in \operatorname{Ann}_{L}(M)$ implies that a subalgebra $D$ is Abelian. Since $\zeta(L)=\langle 0\rangle, L \neq \operatorname{Ann}_{L}(M)$. By Lemma 2.3 in [12], we obtain that $D$ includes an $L$-invariant subalgebra $C$ such that $D=M \oplus C$. Since $D \neq M, C$ is non-zero, and we obtain a contradiction.

Suppose now that $M$ is non-Abelian. Then $A \cap M=\langle 0\rangle$, and, again, we obtain a contradiction. This contradiction shows that $\operatorname{Ann}_{L}(M)=M$.

\section{Proof of Theorem 4}

Let $M$ be a monolith of $L$. By its choice, $M$ is a minimal ideal of $L$. By Proposition 2, $\operatorname{Ann}_{L}(M)=M$. In particular, it follows that $M$ is a maximal Abelian ideal of $L$. Proposition 2 shows also that the factoralgebra $L / M$ is Abelian. It follows that $[x, y] \in M$ for every pairs of elements $x, y \in L$. Since $\langle 0\rangle=M \cap \zeta(L)$, there exists an element $d$ such that $d \notin \operatorname{Ann}_{L}(M)$. Put

$$
[d, M]=\{[d, a] \mid a \in M\} .
$$

Clearly, $[d, M]$ is a subspace of $M$. Since $M$ is Abelian, $[d, M]$ is a subalgebra of $L$. Let $x$ be an arbitrary element of $L$. For each element $a \in A$, we have

$$
[[d, a], x]+[[x, d], a]+[[a, x], d]=0 \text { or }[[d, a], x]=-[[a, x], d]=[d,[a, x]] .
$$

By the above remark, $[x, d] \in M$. Therefore, $[[x, d], a]=0$, because $M$ is Abelian. Thus, we obtain $[[d, a], x]+[[a, x], d]=0$ or $[[d, a], x]=$ $-[[a, x], d]=[d,[a, x]]$. Since $M$ is an ideal, $[a, x] \in M$, so that $[d,[a, x]] \in$ $[d, M]$, and $[[d, a], x] \in M$. This means that $[d, M]$ is an ideal of $L$.

The choice of $d$ shows that $[d, M]$ is a non-zero ideal. Since $M$ is a minimal ideal, we obtain $M=[d, M]$.

Let again $x$ be an arbitrary element of $L$. As we noted above, $[d, x] \in M$. The equality $M=[d, M]$ shows that there exists an element $b \in M$ such that $[d, x]=[d, b]$. It follows that $[d, x-b]=0$, so that $x-b=c \in \operatorname{Ann}_{L}(d)$. Then $x=b+c \in M+A$, where $A=\operatorname{Ann}_{L}(d)$.

Let $a \in A \cap M=\operatorname{Ann}_{M}(d)$. For an arbitrary element $x \in L$, we have

$$
[[a, x], d]+[[d, a], x]+[[x, d], a]=0 .
$$

The choice of $a$ shows that $[d, a]=0$. By the above remark, $[x, d] \in$ $M$. Therefore, $[[x, d], a]=0$, because $M$ is Abelian. Thus, we obtain 
$[[a, x], d]=0$, and this implies that $\operatorname{Ann}_{M}(d)$ is an ideal of $L$. The choice of an element $d$ shows that the equality $\operatorname{Ann}_{M}(d)=M$ is impossible, so that $\langle 0\rangle=\operatorname{Ann}_{M}(d)=A \cap M$.

The equality $L=M \oplus C$ implies that $d=c+b$ for some elements $c \in C, b \in M$. Then $c=d-b$. If $a$ is an arbitrary element of $M$, then the equality $M=[d, M]$ implies that there is an element $v \in M$ such that $-b=[d, v]$. It follows that $c=d+[d, v]$, that is, $c=\mathbf{c}_{v}(d)$. As we have noted above, the mapping $\mathbf{c}_{v}$ is linear. Let $x, y$ be arbitrary elements of $L$. We have

$$
\mathbf{c}_{v}([x, y])=[x, y]+[[x, y], v]=[x, y],
$$

because $[x, y] \in M$, and the ideal $M$ is Abelian. Further, we have

$$
\begin{aligned}
{\left[\mathbf{c}_{v}(x), \mathbf{c}_{v}(y)\right] } & =[x+[x, v], y+[y, v]] \\
& =[x, y]+[[x, v], y]+[x,[y, v]]+[[x, v],[y, v]] \\
& =[x, y]+[[x, v], y]+[x,[y, v]]
\end{aligned}
$$

and

$$
[[x, v], y]+[[y, x], v]+[[v, y], x]=0 .
$$

Again, $[[y, x], v]=0$. So, we obtain

$$
[[x, v], y]+[[v, y], x]=0
$$

or

$$
[[x, v], y]=-[[v, y], x]=[x,[v, y]]=-[x,[y, v]] .
$$

It follows that $\left[\mathbf{c}_{v}(x), \mathbf{c}_{v}(y)\right]=[x, y]=\mathbf{c}_{v}([x, y])$. Hence, the mapping $\mathbf{c}_{v}$ is a homomorphism.

Let $x, y$ be two arbitrary elements of $L$. Suppose that $x \neq y$. If $x, y \in M$, then $\mathbf{c}_{v}(x)=x+[x, v]=x \neq y=y+[y, v]=\mathbf{c}_{v}(y)$. If $x \in M, y \notin M$, then $\mathbf{c}_{v}(x)=x \in M, \mathbf{c}_{v}(y)=y+[y, v] \notin M$, so that $\mathbf{c}_{v}(x) \neq \mathbf{c}_{v}(y)$. Finally, let $x, y \notin M$. We have $x=u_{1}+b_{1}, y=u_{2}+b_{2}$, where $u_{1}, u_{2} \in M, b_{1}, b_{2} \in A$. Then $[x, v]=\left[b_{1}, v\right],[y, v]=\left[b_{2}, v\right]$. Suppose that $\mathbf{c}_{v}(x)=\mathbf{c}_{v}(y)$, i.e.,

$$
x+[x, y]=u_{1}+b_{1}+\left[b_{1}, v\right]=y+[y, v]=u_{2}+b_{2}+\left[b_{2}, v\right] .
$$

It follows that $b_{1}-b_{2}=u_{2}-u_{1}+\left[b_{2}, v\right]-\left[b_{1}, v\right]$. The equality $A \cap M=\langle 0\rangle$ implies that $b_{1}-b_{2}=0$ and $b_{1}=b_{2}$. Then $\left[b_{1}, v\right]=\left[b_{2}, v\right]$, and we obtain that $u_{2}-u_{1}=0$ and $u_{1}=u_{2}$. Thus, $x=y$, and we obtain a contradiction, which proves that $\mathbf{c}_{v}(x) \neq \mathbf{c}_{v}(y)$. Hence, the mapping $\mathbf{c}_{v}$ is a monomorphism. 
Let $x$ be an arbitrary element of $L, x=u_{1}+b_{1}$, where $u_{1} \in M, b_{1} \in A$, and $y=b_{1}+u_{1}-\left[b_{1}, v\right]$. Then

$$
\begin{aligned}
\mathbf{c}_{v}(y) & =y+[y, v]=b_{1}+u_{1}-\left[b_{1}, v\right]+\left[b_{1}+u 1-[b 1, v], v\right] \\
& =b_{1}+u_{1}-\left[b_{1}, v\right]+\left[b_{1}, v\right]=b_{1}+u_{1}=x .
\end{aligned}
$$

Hence, the mapping $\mathbf{c}_{v}$ is an epimorphism and, therefore, an automorphism. We get

$$
L=\mathbf{c}_{v}(L)=\mathbf{c}_{v}(M) \oplus \mathbf{c}_{v}(A)=M \oplus \mathbf{c}_{v}(A) .
$$

If $x \in A$, then $[d, a]=0$ and $\left[c, \mathbf{c}_{v}(x)\right]=\left[\mathbf{c}_{v}(d), \mathbf{c}_{v}(x)\right]=[d, x]=0$. It follows that $\mathbf{c}_{v}(A) \leqslant \operatorname{Ann}_{L}(c)$. In turn, it follows that $\operatorname{Ann}_{L}(c)=$ $\mathbf{c}_{v}(A) \oplus\left(M \cap \operatorname{Ann}_{L}(c)\right)$. Clearly, $[c, M]=[d, M]$. It follows that $\langle 0\rangle=$ $\operatorname{Ann}_{M}(c)=M \cap \operatorname{Ann}_{L}(c)$. Thus, $\operatorname{Ann}_{L}(c)=\mathbf{c}_{v}(A)$. Since the subalgebra $C$ is Abelian, $C \leqslant \operatorname{Ann}_{L}(c)$. This inclusion together with the equality $L=M \oplus \operatorname{Ann}_{L}(c)$ imply that $C=\operatorname{Ann}_{L}(c)=\mathbf{c}_{v}(A)$.

\section{References}

[1] A.M. Bloh, On a generalization of the concept of Lie algebra, Dokl. AN SSSR, N.165, 1965, pp. 471-473.

[2] A.M. Bloh, Cartan-Eilenberg homology theory for a generalized class of Lie algebras, Dokl. AN SSSR, N.175, 1967, pp. 266-268.

[3] A.M. Bloh, A certain generalization of the concept of Lie algebra, Moskov. Gos. Ped. Inst., Uch. Zap., N.375, 1971, pp. 9-20.

[4] J.-L. Loday, Une version non commutative des algebres de Lie; les algebras de Leibniz, Enseign. Math., N.39, 1993, pp. 269-293.

[5] J.-L. Loday,, Cyclic homology, Grundlehren der Mathematischen Wissenschaften, Vol. 301, 2nd ed., Springer, Berlin, 1998.

[6] V.A. Chupordia, L.A. Kurdachenko, I.Ya. Subbotin, On some "minimal" Leibniz algebras, Journal of Algebra and its Application, 16, N.2, 2017.

[7] L.A. Kurdachenko, N.N. Semko, I.Ya. Subbotin, The Leibniz algebras whose subalgebras are ideals, Open Math., N.15, 2017, pp. 92-100.

[8] L.A. Kurdachenko, N.N. Semko, I.Ya. Subbotin, The Leibniz algebras whose subalgebras are ideals, Dopov. Nac. akad. nauk. Ukr., N.6, 2017, pp.9-13.

[9] L.A. Kurdachenko, J. Otal, A.A. Pypka, Relationships between factors of canonical central series of Leibniz algebras, European Journal of Mathematics, N.2, 2016, pp. 565-577.

[10] L.A. Kurdachenko, N.N. Semko, I.Ya. Subbotin, From groups to Leibniz algebras: Common approaches, parallel results, Advances in Group Theory and Applications, N.5, 2018, pp. 1-31.

[11] L.A. Kurdachenko, J. Otal, I.Ya. Subbotin, On some properties of the upper central series in Leibniz algebras, Comment. Math. Univ. Carolin., 60, N.2, 2019, pp. 161-175.

[12] L.A. Kurdachenko, A.A. Pypka, I.Ya. Subbotin, On some relations between the factors of the upper and lower central series in Lie algebras, Serdica Math. J., N.41, 2015, pp. 293-306. 


\section{CONTACT INFORMATION}

Vasyl A. Chupordia, Oles Honchar Dnipro National University, Leonid A. Kurdachenko 72 Gagarin avenue, 49010, Dnipro, Ukraine E-Mail(s): vchupordia@gmail.com, lkurdachenko@i.ua

Nikolaj N. Semko University of the State Fiscal Service of Ukraine, 31 Universitetskaya str., 08205, Irpin, Ukraine.

E-Mail(s): dr.mykola.semko@gmail.com

Received by the editors: 22.01.2020. 